Acta Crystallographica Section D

Biological

Crystallography

ISSN 0907-4449

Yang Li, Yujiong He and Yu Luo*

Department of Biochemistry, University of Saskatchewan, A3 Health Sciences Building, 107 Wiggins Road, Saskatoon,

Saskatchewan S7N 5E5, Canada

Correspondence e-mail: yu.luo@usask.ca

\title{
Conservation of a conformational switch in RadA recombinase from Methanococcus maripaludis
}

Archaeal RadAs are close homologues of eukaryal Rad51s ( $\sim 40 \%$ sequence identity). These recombinases promote ATP hydrolysis and a hallmark strand-exchange reaction between homologous single-stranded and double-stranded DNA substrates. Pairing of the $3^{\prime}$-overhangs located at the damaged DNA with a homologous double-stranded DNA enables the re-synthesis of the damaged region using the homologous DNA as the template. In recent studies, conformational changes in the DNA-interacting regions of Methanococcus voltae RadA have been correlated with the presence of activity-stimulating potassium or calcium ions in the ATPase centre. The series of crystal structures of $M$. maripaludis RadA presented here further suggest the conservation of an allosteric switch in the ATPase centre which controls the conformational status of DNA-interacting loops. Structural comparison with the distant Escherichia coli RecA homologue supports the notion that the conserved Lys248 and Lys250 residues in RecA play a role similar to that of cations in RadA. The conservation of a cationic bridge between the DNAinteracting L2 region and the terminal phosphate of ATP, together with the apparent stability of the nucleoprotein filament, suggests a gap-displacement model which may explain the advantage of ATP hydrolysis for DNA-strand exchange.

\section{Introduction}

The bacterial RecA (Clark \& Margulies, 1965), archaeal RadA (Sandler et al., 1996) and eukaryal Rad51 (Shinohara et al., 1992) and DMC1 (Bishop et al., 1992) proteins, which belong to a recombinase superfamily (Seitz \& Kowalczykowski, 2000), facilitate a central DNA strand-exchange process between a single-stranded DNA (ssDNA) and a homologous double-stranded DNA (dsDNA) in homologous recombination. This recombinase-facilitated process is essentially identical to the denaturing and annealing steps in a PCR reaction except that no temperature manipulation is required. Homologous recombination appears to be essential by playing a pivotal role in the repair of double-stranded DNA breaks and the restarting of stalled replication forks (Cox, 1998; Cox et al., 2000; Courcelle et al., 2001; Lusetti \& Cox, 2002; Kowalczykowski, 2000). Despite large differences between the bacterial and nonbacterial recombinases in their primary structures (less than $30 \%$ sequence identity), electron-microscopic and crystallographic results have revealed strikingly similar 'active' recombinase assemblies (VanLoock et al., 2003; Conway et al., 2004; Wu et al., 2004; Chen et al., 2008; Sheridan
Received 2 March 2009

Accepted 30 March 2009

PDB References: RadA, 3etl, r3etlsf; 3ew9, r3ew9sf; 3ewa, r3ewasf. 
et al., 2008). The structures of EcRecA in complex with a series of DNA molecules have shed light on the exact mechanism of homologous DNA-strand exchange (Chen et al., 2008). These right-handed helical recombinase assemblies are classic allosteric systems equipped with two functional sites: one located at the subunit interface for binding and hydrolyzing ATP and the other near the filament axis for binding DNA and promoting strand exchange. In the very first crystal structure of EcRecA in complex with ADP, the DNA-binding regions L1 and L2 are disordered in an apparently inactive form (Story et al., 1992). The L1 and L2 regions of other crystallized inactive filaments of bacterial RecAs are either disordered (Datta et al., 2000; Rajan \& Bell, 2004; Xing \& Bell, $2004 a, b$ ) or observed in an ensemble of conformations (Datta, Ganesh et al., 2003; Datta, Krishna et al., 2003; Krishna et al., 2007). It is increasingly clear that the conformations of these loops are controlled by allosteric effectors such as the nucleotide cofactor (Datta, Ganesh et al., 2003; Datta, Krishna et al., 2003; Krishna et al., 2007). Strikingly, these loops become highly ordered around the bound DNA in the recently crystallized active form of EcRecA in complex with ADP-AlF and DNA (Chen et al., 2008). Similar conformational changes of the DNA-binding regions have also been observed in crystal structures of MvRadA (Wu et al., 2005; Qian, He, Ma et al., 2006). The cation-dependence of RadA enabled us to propose the hypothesis of the existence of a conformational switch in the ATPase centre (Wu et al., 2005). Since such cation-dependence appears to be ubiquitous in the closely related group of archaeal RadA (Wu et al., 2005; Qian, He, Wu et al., 2006; Qian, He, Ma et al., 2006), eukaryal Rad51 (Rice et al., 2001; Liu et al., 2004; Sehorn et al., 2004; Shim et al., 2006; Bugreev \& Mazin, 2004) and DMC1 (Bugreev et al., 2005; Lee et al., 2005) proteins, it should be possible to observe concerted conformational changes in another homologue. We set out to study conformational changes in several RadA recombinases. MmRadA, a close homologue of MvRadA ( $\sim 81 \%$ sequence identity), turned out to be amenable to crystallization. Similar to MvRadA, crystallized MmRadA has been observed in two sets of conformations correlated with the presence or absence of a stimulating concentration of ammonium or potassium ions. Structural comparison with its distant EcRecA homologue supports the notion that the two invariant lysine residues (residues 248 and 250 in EcRecA) in bacterial RecAs play a role similar to that of monovalent cations in RadA (Wu et al., 2005; Qian, He, Wu et al., 2006).

\section{Materials and methods}

\subsection{Cloning, protein preparation and crystallization}

The open reading frame of RadA from M. maripaludis was inserted between the NcoI and XhoI sites of pET28a (Nova-

\footnotetext{
${ }^{1}$ Abbreviations: MmRadA, RadA recombinase from Methanococcus maripaludis; MvRadA, RadA recombinase from M. voltae; EcRecA, RecA recombinase from Escherichia coli; ssDNA, single-stranded DNA; dsDNA, double-stranded DNA; hdDNA, heteroduplex DNA; ATP, adenosine 5'triphosphate; AMPPNP, 5'-adenylyl imidodiphosphate, a nonhydrolyzable analogue of ATP; FAM, fluorescein phosphoamidite.
}

gen). The resulting plasmid was verified by DNA sequencing using $\mathrm{T} 7$ promoter and terminator primers. The recombinant MmRadA was overexpressed in BL21 Rosetta2 (DE3) cells (Novagen) and purified as reported for MvRadA (Wu et al., 2004; Qian, He, Wu et al., 2006). In brief, the purification procedure involved steps of polymin P (Sigma) precipitation and high-salt extraction and three chromatographic steps using heparin (Amersham Biosciences), hydroxyapatite (BioRad) and DE52 anion-exchange (Whatman) columns. The purified MvRadA protein was concentrated to $\sim 30 \mathrm{mg} \mathrm{ml}^{-1}$ by ultrafiltration.

\subsection{ATPase assay}

A solution containing $0.033 \%(w / v)$ Malachite Green, $1.3 \%(w / v)$ ammonium molybdate and $1.0 \mathrm{M} \mathrm{HCl}$ was used to monitor the release of inorganic phosphate (Itaya \& Ui, 1966) by ATP hydrolysis. The absorbance at $620 \mathrm{~nm}$ was recorded for quantification. The reaction solutions for the ssDNAdependent ATPase assay contained $3 \mu M$ RadA, $18 \mu M$ ssDNA (in nucleotides), $5 \mathrm{~m} M$ ATP, $0.05 M$ Tris-HEPES buffer $\mathrm{pH} 7.4,100 \mathrm{mM}$ of a specified monovalent salt, $10 \mathrm{mM}$ $\mathrm{MgCl}_{2}$ and $0.1 \%(v / v)$ 2-mercaptoethanol. The 36-nucleotide oligonucleotide poly-(dT) ${ }_{36}$ (Integrated DNA Technologies) was used as the ssDNA substrate. The conditions for the highsalt-stimulated ATPase assay were similar except for the presence of a $1.0 \mathrm{M}$ concentration of a specified monovalent salt as a substitute for the ssDNA.

\subsection{Strand-exchange assay using synthetic oligonucleotides}

The DNA substrates were modified based on a published study (Mazin et al., 2000). Three oligonucleotides (\#FAM43, 43 nucleotides, FAM-TTTTG CGGAT GGCTT AGAGC TTAAT TGCTG AATCT GGTGC TGT; \#45A, 36 nucleotides, ACAGC ACCAG ATTCA GCAAT TAAGC TCTAA GCCATG; \#55A, 36 nucleotides, GATGG CTTAG AGCTT AATTG CTGAA TCTGG TGCTGT) were obtained from Integrated DNA Technologies. Equal molarities of complementary oligonucleotides \#45A and \#55A were heated at $368 \mathrm{~K}$ for $5 \mathrm{~min}$ and then slowly cooled to $294 \mathrm{~K}$ to generate the 36 bp dsDNA substrate. The strand-exchange solution was composed of $5 \mathrm{~m} M$ ATP or an analogous nucleotide, $10 \mathrm{mM}$ $\mathrm{MgCl}_{2}, 100 \mathrm{~m} M$ of a specified monovalent salt, $50 \mathrm{mM}$ HEPES-Tris buffer $\mathrm{pH} 7.4,15 \mu M$ MvRadA, $0.1 \%(v / v)$ 2-mercaptoethanol and $1 \mu M$ oligonucleotides. The 43nucleotide 5'-fluorescein-labelled ssDNA substrate (oligonucleotide \#FAM43) was pre-incubated at $310 \mathrm{~K}$ with MvRadA for 1 min before adding the 36 bp dsDNA substrate. The reaction was stopped after $30 \mathrm{~min}$ by adding EDTA to a concentration of $20 \mathrm{mM}$ and trypsin to a concentration of $1 \mu \mathrm{g} \mu \mathrm{l}^{-1}$. After $10 \mathrm{~min}$ of proteolysis, $10 \mu \mathrm{l}$ sample was mixed with $5 \mu \mathrm{l}$ loading buffer composed of $30 \%$ glycerol and $0.1 \%$ Bromophenol Blue, loaded onto a 17\% SDS-polyacrylamide vertical gel and developed for $1 \mathrm{~h}$ at $100 \mathrm{~V}$. The fluorescent emission by the intrinsic fluorescein was recorded using a Kodak GelLogic 200 system. 
Table 1

Data-collection and refinement statistics.

Values in parentheses are for the highest resolution shell.

\begin{tabular}{|c|c|c|c|}
\hline PDB code & 3etl & 3ew9 & 3ewa \\
\hline Monovalent salt & $0.1 \mathrm{M} \mathrm{NH}_{4} \mathrm{Cl}$ & $0.4 \mathrm{M} \mathrm{KCl}$ & $0.4 \mathrm{M} \mathrm{NH}_{4} \mathrm{Cl}$ \\
\hline \multicolumn{4}{|l|}{ Data collection } \\
\hline Space group & $P 6_{1}$ & $P 6_{1}$ & $P 6_{1}$ \\
\hline \multicolumn{4}{|l|}{ Unit-cell parameters } \\
\hline$a=b(\AA)$ & 81.1 & 81.2 & 81.1 \\
\hline$c(\AA)$ & 108.5 & 107.1 & 107.2 \\
\hline$\alpha=\beta\left({ }^{\circ}\right)$ & 90 & 90 & 90 \\
\hline$\gamma\left({ }^{\circ}\right)$ & 120 & 120 & 120 \\
\hline Resolution $(\AA)$ & $2.4(2.5-2.4)$ & $2.4(2.5-2.4)$ & $2.0(2.1-2.0)$ \\
\hline$R_{\text {merge }}$ & $0.069(0.347)$ & $0.057(0.379)$ & $0.061(0.448)$ \\
\hline$I / \sigma(I)$ & $17.1(4.8)$ & $15.8(3.0)$ & $17.5(3.5)$ \\
\hline Completeness (\%) & $96.8(72.0)$ & $94.6(83.2)$ & $97.6(91.6)$ \\
\hline Unique reflections & 15276 (1033) & 15225 (1237) & $26987(3375)$ \\
\hline Redundancy & $9.9(9.3)$ & $6.8(6.1)$ & $10.7(10.6)$ \\
\hline \multicolumn{4}{|l|}{ Refinement } \\
\hline Resolution (§) & $20-2.4$ & $20-2.4$ & $20-2.0$ \\
\hline No. of reflections & 15276 & 15225 & 26987 \\
\hline$R_{\text {work }} / R_{\text {free }}$ & $0.222 / 0.260$ & $0.216 / 0.260$ & $0.223 / 0.255$ \\
\hline No. of atoms & 2364 & 2449 & 2483 \\
\hline Protein & 2323 & 2406 & 2405 \\
\hline Ligand/ion & 33 & 35 & 33 \\
\hline Water & 8 & 8 & 45 \\
\hline$B$ factor $\left(\AA^{2}\right)$ & 47.1 & 47.5 & 38.5 \\
\hline Protein & 47.2 & 47.6 & 38.7 \\
\hline Ligand/ion & 39.2 & 42.4 & 32.5 \\
\hline Water & 31.8 & 30.4 & 34.8 \\
\hline \multicolumn{4}{|l|}{ R.m.s. deviations } \\
\hline Bond lengths $(\AA)$ & 0.0068 & 0.0071 & 0.0060 \\
\hline Bond angles $\left({ }^{\circ}\right)$ & 1.22 & 1.24 & 1.21 \\
\hline
\end{tabular}

\subsection{Crystallization of MmRadA and diffraction data collection}

Hexagonal MmRadA crystals (space group $P 6_{1}$ ) were grown by the hanging-drop vapour-diffusion method and grew to maximum dimensions of $0.1 \times 0.1 \times 0.3 \mathrm{~mm}$. The protein sample contained $\sim 1 \mathrm{mM}$ concentrated RadA and $2 \mathrm{mM}$ AMPPNP. The reservoir solutions contained $6-8 \%$ polyethylene glycol $3350,50 \mathrm{~m} M \mathrm{MgCl}_{2}, 50 \mathrm{~m} M$ Tris- $\mathrm{HCl}$ buffer $\mathrm{pH} 7.9$ and $0.1-0.4 M$ of a monovalent salt $(\mathrm{NaCl}, \mathrm{KCl}, \mathrm{RbCl}$ or $\mathrm{NH}_{4} \mathrm{Cl}$ ). The crystals were transferred into a stabilizing solution composed of the reservoir solution supplemented with $28 \%(w / v)$ sucrose, looped out of the solution and frozen in a nitrogen cryostream at $100 \mathrm{~K}$. The diffraction data sets were first collected using an in-house Bruker Proteum system at a wavelength of $1.54 \AA$ and subsequently using synchrotron radiation at a wavelength of $0.97 \AA$. The synchrotron data sets were processed using the $X D S$ program (Kabsch, 1993). The statistics of the diffraction data are listed in Table 1.

\subsection{Structural determination and refinement}

The previously solved MvRadA model (PDB code 1t4g) was used as the starting model. Rigid-body refinement using low-resolution data (20-6.0 $)$ ) was initially employed. The models were then iteratively rebuilt using XtalView (McRee, 1999) and refined using CNS (Brünger et al., 1998). Statistics of the refinement and model geometry are also given in Table 1. The molecular figures were generated using $M O L$ -
SCRIPT (Kraulis, 1991) and rendered using RASTER3D (Bacon \& Anderson, 1988). The coordinates and structure factors have been deposited in the Protein Data Bank (PDB codes 3etl, 3ew9 and 3ewa).

\section{Results}

\subsection{DNA-dependent ATPase activity is selective towards monovalent cations}

As reported previously, potassium is the only commonly used monovalent cation which stimulates the ATPase activity of MvRadA (Wu et al., 2005). We first studied the ATPase activity of the MmRadA homologue in the presence of an ssDNA [poly-(dT) $)_{36}$ ] and $100 \mathrm{mM}$ of a monovalent cation $\left(\mathrm{Na}^{+}, \mathrm{NH}_{4}^{+}, \mathrm{Rb}^{+}\right.$or K $\mathrm{K}^{+}$; Fig. $\left.1 a\right)$. An MmRadA:ssDNA ratio of 1:6 (six nucleotides per protein) was used in this assay. As expected from its high level of similarity to MvRadA ( $~ 81 \%$ sequence identity), the M. maripaludis protein also actively catalyzed ATP hydrolysis in the presence of potassium but not of sodium. However, MmRadA appears to be less selective than MvRadA towards monovalent cations. In the presence of the similarly sized ammonium or rubidium ions, the ATPase activities were similar (over $60 \%$ ) to that in the presence of potassium.

\subsection{High-salt-stimulated ATPase activity is selective towards monovalent cations}

Many bacterial RecA proteins, as well as eukaryal $\operatorname{Rad} 51$ and archaeal RadA proteins, are known to become ATPaseactive in the presence of high salt as a substitute for ssDNA (Pugh \& Cox, 1988; Tombline \& Fishel, 2002; Liu et al., 2004; Wu et al., 2005). As expected, MmRadA shares this property in the presence of $1.0 \mathrm{M} \mathrm{KCl}$ or $\mathrm{NH}_{4} \mathrm{Cl}$ (Fig. 1b). Although $\mathrm{RbCl}$ stimulated the ssDNA-dependent ATPase activity of MmRadA, $1.0 \mathrm{M} \mathrm{RbCl}$ did not noticeably stimulate the ATPase activity of MmRadA in the absence of DNA.

\subsection{DNA-strand exchange activity is selective towards monovalent cations}

In the strand-exchange assay (Fig. 2), an MmRadA:ssDNA ratio of 1:3 (three nucleotides per protein) was used, which conforms to the known stoichiometry for such recombinases. In the presence of ATP, the M. maripaludis protein was active for all four monovalent cations tested. In the presence of AMPPNP, however, it was active with only two $\left(\mathrm{K}^{+}\right.$and $\left.\mathrm{NH}_{4}^{+}\right)$ of the four cations.

\subsection{The crystal structure of an ATPase-active conformation of MmRadA}

Since $\mathrm{KCl}$ and $\mathrm{NH}_{4} \mathrm{Cl}$ were observed to stimulate the ATPase activity of MmRadA in the absence of ssDNA as well as the strand-exchange activity of MmRadA in the presence of AMPPNP, we reasoned that both salts could serve as stabilizing reagents for MmRadA to remain predominantly in its ATPase-active conformation. In the presence of $0.4 M$ of 
either salt, MmRadA was indeed crystallized in a largely ordered conformation (except for seven residues from Pro262 to Val268) similar to the ATPase-active conformation seen in MvRadA (Wu et al., 2005; Table 1). The two MmRadA structures are essentially identical except for the higher resolution data obtained for the crystal of MmRadA in the presence of $0.4 \mathrm{M} \mathrm{NH}_{4} \mathrm{Cl}$. All crystals reported in this study were grown under similar conditions apart from the concentration and identity of the monovalent salt. The crystals belong to space group $P 6_{1}$, with similar helical pitches (107.1$108.5 \AA$ ) coinciding with the crystallographic $c$ axis (Table 1 ). Although this packing scheme appears to be similar to that reported for MvRadA (104-107 $\AA$ pitch), the mode of inter-

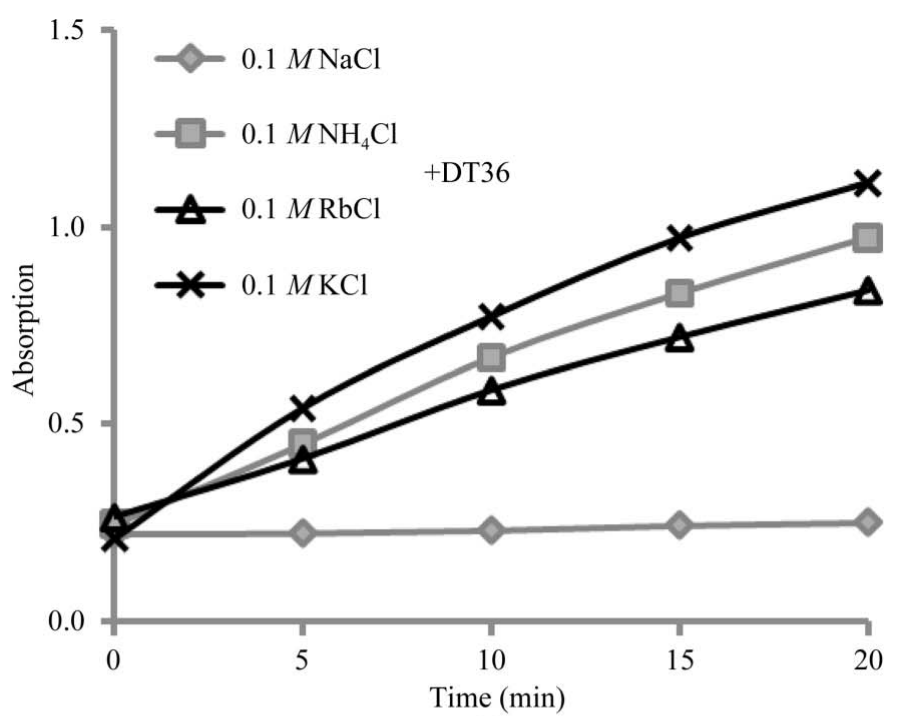

(a)

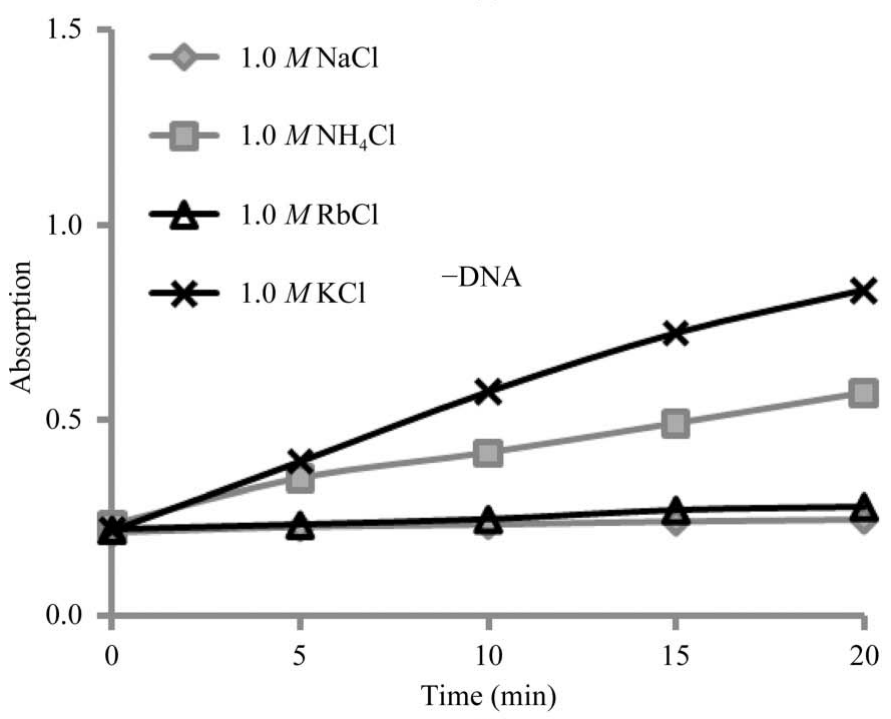

Figure 1

(b)

Monovalent cation-dependence of ATP hydrolysis catalyzed by MmRadA. The reaction solutions contained $5 \mathrm{mM}$ ATP, a specified amount of a monovalent salt, $10 \mathrm{~m} M \mathrm{MgCl}_{2}$ and $50 \mathrm{~m} M$ HEPES-Tris buffer $\mathrm{pH}$ 7.4. The solutions also contained $3 \mu M$ MmRadA. (a) Time courses of phosphate release in the presence of $100 \mathrm{~m} M$ of a specified monovalent salt and $1 \mu M$ poly- $(\mathrm{dT})_{36}$. (b) Time courses in the presence of $1.0 \mathrm{M}$ of a specified monovalent salt. DNA was absent. filament interaction is different. In MvRadA crystals, the crystal packing is stabilized by a repeated mode of cationbridged inter-filament interaction between an $\mathrm{N}$-terminal turn (Thr6-Val11) and Glu164. Three carbonyl groups (Thr6, Leu8 and Val11) and the side chain of Glu164 coordinate the bridging $\mathrm{Na}^{+}$or $\mathrm{K}^{+}$. In MmRadA crystals, the role of the $\mathrm{N}$-terminal turn in inter-filament packing is similar. The cation-bridged interaction, however, is replaced by direct hydrogen bonds between the three carbonyl groups of the $\mathrm{N}$-terminal turn and the side chain of Lys318. The helical pitch ranges from 107.1 to $107.2 \AA$ in this ATPase-active form obtained in the presence of $0.4 \mathrm{M} \mathrm{KCl}$ or $\mathrm{NH}_{4} \mathrm{Cl}$. The nonhydrolyzable ATP analogue AMPPNP is buried between the MmRadA monomers as expected. An OMIT difference electron-density map of the crystal in the presence of $0.4 M$ $\mathrm{NH}_{4} \mathrm{Cl}$ revealed two solvent molecules near the $\gamma$-phosphate of the ATP analogue (Fig. $3 a$ ). These are probably ammonium ions, since both are located at positions corresponding to the potassium ions in the structure with $0.4 \mathrm{M} \mathrm{KCl} \mathrm{(map} \mathrm{not}$ shown). The twin ions are likely to provide polarization of the $\gamma$-phosphate in addition to the electron-withdrawing effects by the well known P-loop and magnesium ion (Saraste et al.,

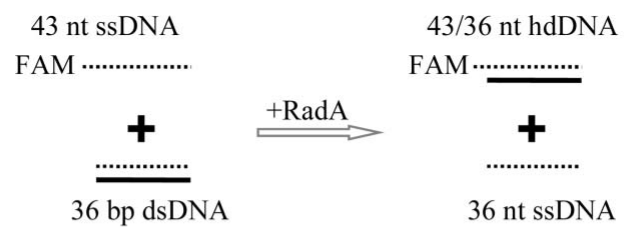

(a)

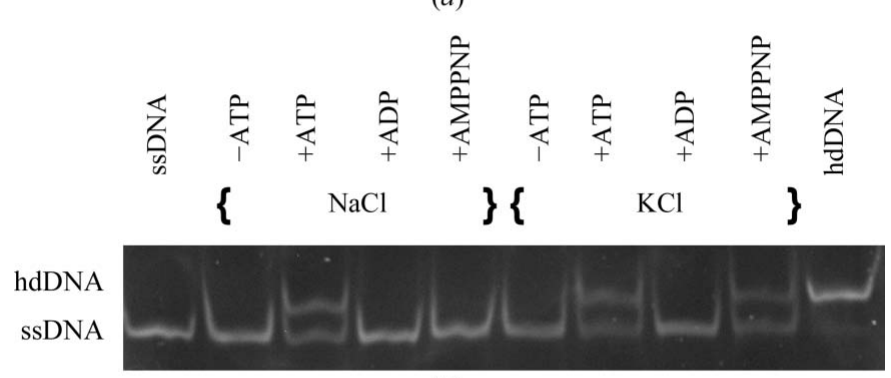

(b)

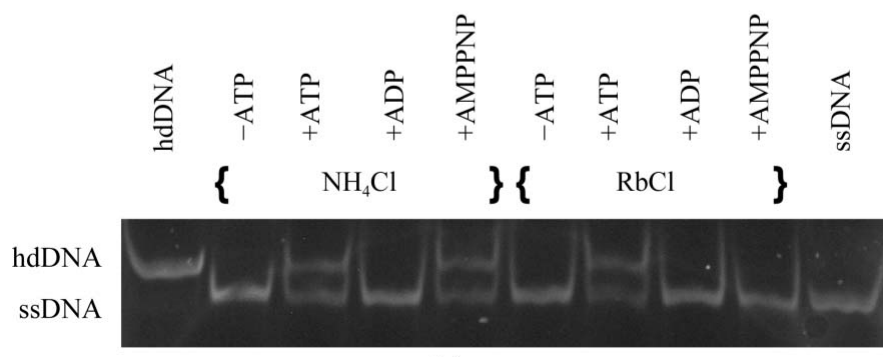

(c)

Figure 2

Monovalent cation-dependence of the DNA strand-exchange activity of MmRadA. The strand reaction scheme is shown in $(a)$. The reaction solutions contained $2 \mathrm{~m} M$ of a specified nucleotide, $100 \mathrm{~m} M$ of a specified monovalent salt, $10 \mathrm{~m} M \mathrm{MgCl}_{2}$ and $50 \mathrm{~m} M$ HEPES-Tris buffer $\mathrm{pH}$ 7.4. The solutions also contained $15 \mu M \mathrm{MmRadA}$ and $1 \mu M$ each of ssDNA (FAM-labelled) and dsDNA (unlabelled) substrates. Strand-exchange activity was indicated by the formation of the slowest migrating heteroduplex DNA species (FAM-labelled hdDNA). The intrinsic fluorescence from the FAM label was recorded as shown in $(b)$ and $(c)$. 
1990), therefore suggesting compatibility of this conformation with requirement for efficient ATP hydrolysis. As seen in the ATPase-active conformation of MvRadA, the MmRadA structure is also highly ordered in its DNA-binding L1 and L2 region (in magenta; Fig. 3b). Noticeably, an eight-residue helix (residues Gly275-Ala282) analogous to helix $G$ of EcRecA (Story et al., 1992) is ordered in the $\mathrm{L} 2$ region. Two $\mathrm{K}^{+} / \mathrm{NH}_{4}^{+}$ ions (yellow spheres; Fig. 3b) form bridges between the $\gamma$-phosphate and the backbone carbonyl moieties at the $\mathrm{C}$-terminus of the eight-residue helix. In addition, the side chain of His 280 makes a direct hydrogen bond to the $\gamma$-phosphate. The root-mean-square deviation between the ATPase-active conformations of MvRadA (PDB code 2fpm) and MmRadA is $0.58 \AA$ for 311 superimposed $\mathrm{C}^{\alpha}$ atoms. The positions of the twin potassium/ammonium ions are essentially identical.

\subsection{The crystal structure of MmRadA in an inactive conformation}

The second set of MmRadA-AMPPNP structures were determined using crystals in the presence of $0.1-0.4 \mathrm{M} \mathrm{NaCl}$ or $\mathrm{RbCl}$ or $0.1-0.3 \mathrm{M} \mathrm{KCl}$ or $\mathrm{NH}_{4} \mathrm{Cl}$. These conditions were selected because they did not appear to provide adequate cationic stabilization to trap the protein in its ATPase-active conformation. Indeed, less ordered structures that were essentially identical to each other were observed. The highest resolution data are listed in Table 1 . In these structures, no plausible ions were seen even for $\mathrm{Rb}^{+}$, which is an electronrich anomalous scatterer. As such, the catalyzing effect of the monovalent cations seen in the ATPase-active conformation is lost in this second set of MmRadA structures. Compared with the ATPase-active filaments of MmRadA, the filament pitch is slightly longer $(108.5 \AA)$. Since very similar filament pitches were observed for multiple data sets under similar ionic conditions (within $0.2 \AA$ ), the difference in helical pitches appeared to be significant. Although the DNA-binding L1 region remains ordered, the L2 region becomes largely disordered (shown in cyan in Fig. $3 b$ ). There was no electron density for 19 residues from Ala260 to Val278. The root-meansquare deviation between this second conformation of MmRadA and the previously reported ATPase-inactive conformation of MvRadA (PDB code $1 \mathrm{t} 4 \mathrm{~g}$ ) is $0.64 \AA$ for 299 superimposed $\mathrm{C}^{\alpha}$ atoms, thus confirming the reoccurrence of a similar inactive conformation in MmRadA.

\section{Discussion}

\subsection{Comparison with electron-microscopy-derived filament parameters}

MmRadA is the second strand-exchange protein of the $\operatorname{RadA} / \operatorname{Rad} 51 / D M C 1$ group to have been crystallized in a form resembling the active filament. Despite its high level of sequence similarity to MvRadA, differences in crystal packing suggest that stabilizing interactions in the crystal lattice did not dictate the conformations observed. As in MvRadA, two sets of conformations have been observed, each resembling the corresponding set in MvRadA. We have conducted soaking experiments with MmRadA crystals. It appears that the conformation is solely determined by the chemical composition of the soaking solution and not by the initial crystallizing solution. Therefore, the ATPase centre and DNA-binding loops of MmRadA are likely to remain mobile in the crystal lattice, which resembles the state in solution. The values of helical pitch and number of subunits per turn in the recently determined structure of active EcRecA filament in complex with DNA $(\sim 94.0 \pm 1.5 \AA$ and $6.16 \pm 0.03$ subunits per turn; Chen et al., 2008) are in close agreement with those derived from electron microscopy ( $96 \AA$ pitch and 6.2 subunits per turn on average; Sheridan et al., 2008). On the other hand, electron microscopy revealed that active filaments of eukaryal Rad51 and DMC1 proteins have $\sim 6.5$ subunits per turn and average pitches ranging from 101 to $103 \AA$ (Sheridan et al., 2008). As a close homologue of the eukaryal strand-exchange proteins, MvRadA filaments formed in the presence of ADP$\mathrm{AlF}_{4}$ have a slightly shorter average pitch of $\sim 99 \AA$ and $\sim 6.25$ subunits per turn (Galkin et al., 2006). The crystal structures of the high-salt-stabilized ATPase-active form of MvRadA have an average pitch of $\sim 105 \AA$ and are apparently overwound, with exactly six subunits per turn. The crystallized ATPaseactive filaments of MmRadA, which are similarly overwound to MvRadA owing to crystal packing along the crystallographic sixfold axis, have even longer pitches of around $107 \AA$. The longer pitch as well as the overwound nature is indicative of the differences between the active recombinase/DNA filament and the high-salt-stabilized ATPase-active forms of RadA recombinases, the latter of which appears to be more flexible and hence amenable to crystallization in space group $P 6_{1}$. Although the loop 2-anchoring helix $G$ in the $\operatorname{RadA}$ structures appears to be similarly positioned to its counterpart in the EcRecA-DNA complex, as suggested by similar orientations of His 280 and its equivalent Phe217 residue located at the C-terminus of this short helix, the overwound nature of the crystallized MvRadA and MmRadA filaments appeared to be incompatible with DNA binding. In fact, efforts to cocrystallize the two RadA proteins with DNA under similar conditions have been futile.

\subsection{Structural comparison of the ATPase centres of MmRadA and EcRecA}

The structure of the DNA-bound active form of EcRecA has recently been elucidated (Chen et al., 2008). Since the RecA proteins are remote homologues of RadA with barely $30 \%$ sequence identity, it would be more meaningful to superimpose the conserved P-loops of EcRecA (PDB code 3cmx; residues 3066-3073) and MmRadA (residues 105-112). The resulting root-mean-square deviation between the 32 equivalent main-chain atoms of the P-loops is $0.30 \AA$. It is not surprising that the P-loop-wrapped triphosphate parts of the two structures are also placed in close vicinity (Fig. 4). The ribose and base counterparts, on the other hand, do not superimpose well. One noticeable difference in the ATPinteracting cap (equivalent to residues 302-308 of MvRadA) is 
that RecA proteins have two conserved lysyl residues (residues 248 and 250 in EcRecA), while $\operatorname{RadA} / \operatorname{Rad} 51 / \mathrm{DMC1}$ proteins share an aspartyl residue (residue 302 in MmRadA and MvRadA). The tip of the side chain of the conserved Lys 248 of EcRecA is placed $\sim 0.7 \AA$ from the cation held in place by Asp302 of MmRadA. This lysyl side chain also forms a hydrogen bond to the carbonyl of Phe217 located at the C-terminal end of helix $G$. In both $\operatorname{RadA}$ proteins the monovalent cation is coordinated by the carbonyl of His 280 at the position equivalent to Phe 217 of RecA. The other conserved lysyl residue in EcRecA, Lys250, is $\sim 2.7 \AA$ from the second monovalent cation in the ATPase centre of RadA.
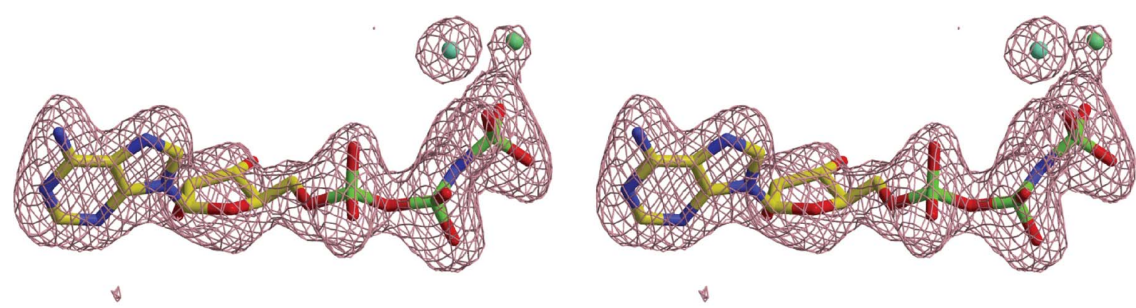

(a)
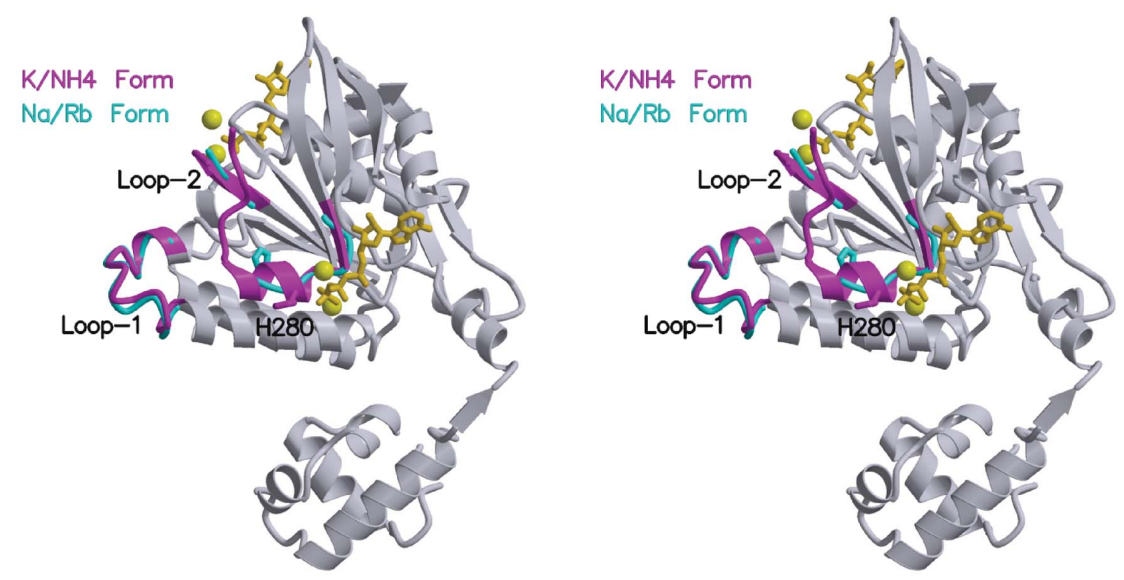

(b)

Figure 3

Crystal structure of MmRadA in complex with AMPPNP in stereo. (a) OMIT $\left(F_{\mathrm{o}}-F_{\mathrm{c}}\right)$ difference Fourier map at $2.0 \AA$ resolution. The AMPPNP molecule is shown as a stick model. The possible locations of two ammonium ions are shown as cyan spheres. (b) Two conformational forms of MmRadA. Two AMPPNP molecules and the side chains of His 280 are shown as stick models. The protein is shown in ribbon representation. The DNAinteracting loops are highlighted in cyan for the recurrent inactive form observed in $\mathrm{NaCl}$, $\mathrm{RbCl}$ or a low concentration $(<0.3 \mathrm{M})$ of $\mathrm{KCl}$ or $\mathrm{NH}_{4} \mathrm{Cl}$. They are highlighted in magenta for the ATPase-active form observed in $0.4 \mathrm{M} \mathrm{KCl}$ or $\mathrm{NH}_{4} \mathrm{Cl}$.
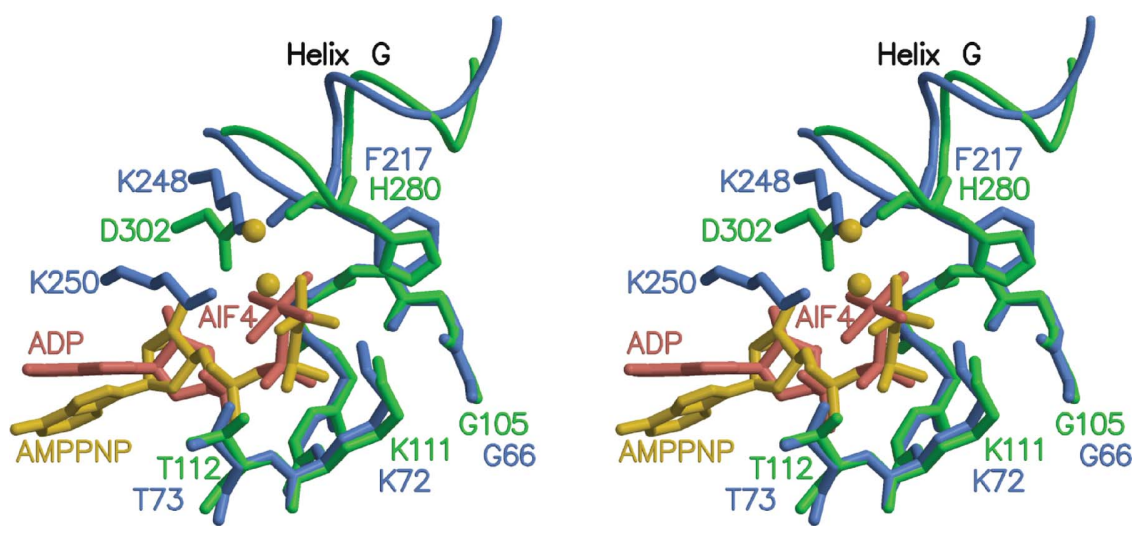

Figure 4

Superimposed ATPase centres of MmRadA and EcRecA in stereo. The conserved P-loops are superimposed. The P-loop (Gly105-Thr112), His280 and Asp302 of the ATPase-active form of MmRadA are shown as green stick models. The MmRadA-bound AMPPNP and monovalent cations are shown as yellow ball-and-stick models. The P-loop (Gly66-Thr73), Phe217, Lys248 and Lys 250 of the active EcRecA structure are shown in blue.

\subsection{Conservation of a conformational switch in the ATPase centres}

The obvious structural similarity between the ATPase centres of RadA and RecA proteins appears to suggest the existence of a conserved cationic bridge between the $\gamma$-phosphate of ATP and the C-terminus of helix $G$ (Fig. 4) which serves as a conformational switch controlled by the ATPhydrolysis cycle. In the recently reported crystal structures of the active forms of MvRadA and EcRecA, this bridge appears to stabilize a highly ordered conformation of the DNA-interacting loop 2 that is compatible with a high-affinity state for DNA binding. ATP hydrolysis would almost certainly lead to the collapse of this stabilizing bridge and result in mobility in loop 2 and hence lower affinity for DNA. Such structural consequences have been observed in the MvRadA-ADP complex (Qian et al., 2005), which is essentially similar to the inactive forms observed in the MvRadA-AMPPNP and MmRadAAMPPNP complexes. Similar consequences could also occur for RecA proteins in the aftermath of ATP hydrolysis. Importantly, ATP hydrolysis does not necessarily lead to filament dissociation, although the inactive form appears to be adaptive to a wider range of helical pitches as seen in the much shorter pitched EcRecA (Story et al., 1992; $28 \AA$ pitch) and MvRadA devoid of its N-terminal domain $(\sim 90 \AA$ pitch; Galkin et al., 2006).

\subsection{Putative advantage of ATP hydrolysis for DNA strand exchange}

The functional importance of ATP hydrolysis has always been an issue of debate for RecA-like strand-exchange proteins since the three-stranded exchange process can also be carried out in the absence of ATP hydrolysis (Kowalczykowski \& Krupp, 1995; Sung \& Stratton, 1996). Three models have been proposed, as reviewed by Cox (2007). Although these models can explain most features of RecA activity, inconsistencies with the known 
structural features of the recombinase assembly exist. The filament-dissociation model and RecA-redistribution model are largely based on hydrolysis-triggered RecA dissociation at the $5^{\prime}$-end or in the interior of the filament. Owing to massive stabilizing interactions between adjacent subunits within the

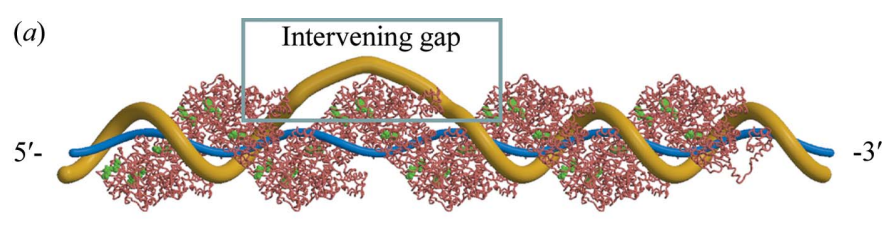

ATP hydrolysis and dsDNA release
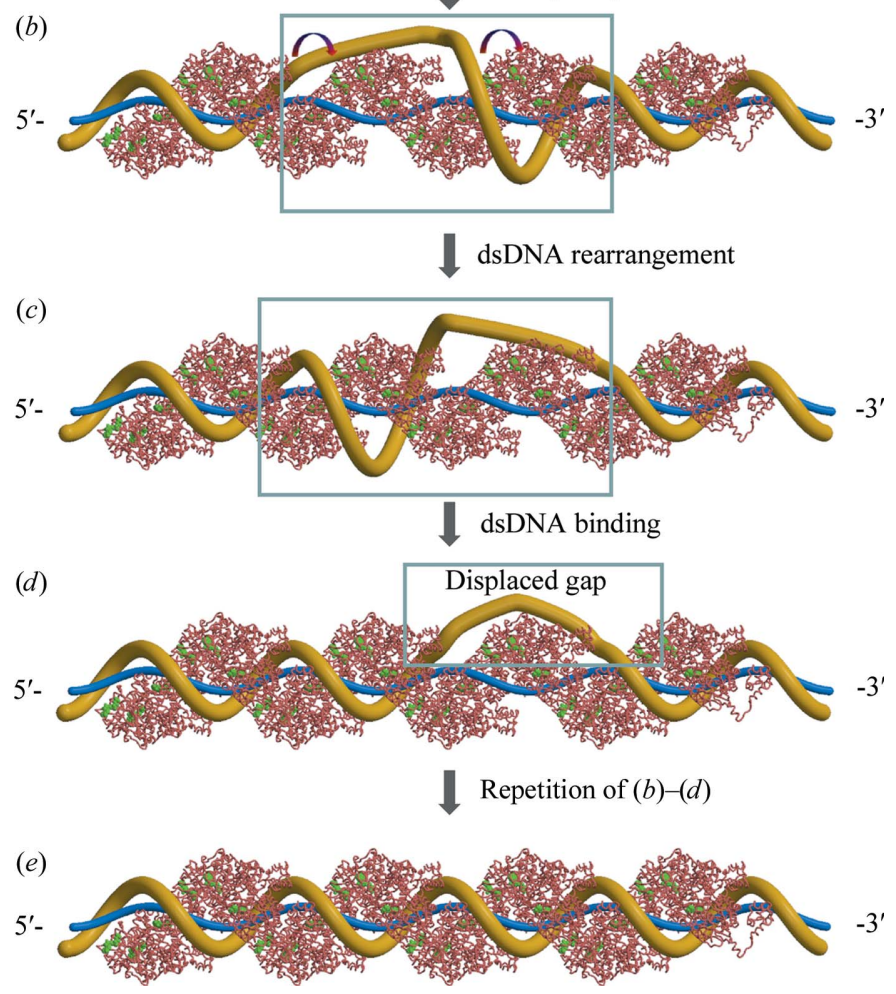

Figure 5

Hypothetical model of ATP hydrolysis-facilitated gap displacement. The crystallized protein filament is shown as a $\mathrm{C}^{\alpha}$ trace in salmon with AMPPNP in green. Speculative models of the DNA substrates are shown as wires. Important segments are boxed. The $5^{\prime}$ - and $3^{\prime}$-ends are based on the filament-initiating ssDNA (thinner wire in blue). The homologous dsDNA is shown as a thicker wire in yellow. The strand-exchange process progresses from the $5^{\prime}$-end to the $3^{\prime}$-end. (a) An intervening gap. Such gaps are likely to exist owing to simultaneous homologous pairing between the recombinase/ssDNA filament and dsDNA at multiple locations. The dsDNA in the gap region cannot become properly wound ( $\sim 19$ bp per helical turn) around the nucleoprotein filament without unwinding its adjacent region(s). Despite the sequence homology, it serves as a topological roadblock of strand exchange between long DNA substrates. (b) ATP hydrolysis promotes the transient release of a dsDNA segment at the immediate $3^{\prime}$-flank of the gap. The transiently released dsDNA region is shown as an exaggerated wide helix. (c) Rearrangement in the transiently released dsDNA region and the adjacent gap takes place without changing the overall topology. The $5^{\prime}$-end of the gap region becomes properly wound, while the released dsDNA region becomes unwound. $(d)$ The rearranged $5^{\prime}$-end of the gap becomes bound by the recombinase filament. As a result, the gap is displaced towards the $3^{\prime}$-end. (e) Repetition of steps $(b)-(d)$ would chase the topologically strained gap out of the $3^{\prime}$-end of the nucleoprotein filament, therefore removing topological roadblocks to extensive DNA strand exchange. filament, the dissociation of recombinase subunits should be minimal except for the filament ends. At the $5^{\prime}$-end of the nucleoprotein, filament dissociation would be blocked by recombinase-recruiting proteins such as bacterial RecFOR (Morimatsu \& Kowalczykowski, 2003) and eukaryal BRCA2 (Yang et al., 2005). Besides, direct observation of filament formation of RecA on dsDNA suggests that net growth at both ends is possible (Galletto et al., 2006). Coupling of the ATP-hydrolytic cycle between adjacent RecA monomers is consistent with the observed filament-dissociation rate at the $5^{\prime}$-end and supports the third model of facilitated DNA rotation (Cox, 2003; Cox et al., 2005). This model has provided an interpretation of how RecA clears unpaired segments by synchronized rotation of these segments. The available crystal structures of RecA-like proteins appear to suggest that ATP hydrolysis would lead to reduced affinity for DNA owing to conformational change in loop 2 and shortened pitch owing to rearrangement at the ATP-binding interface. However, there is no structural evidence to associate a motoring function with RecA-like proteins. The inconsistencies of the three models with the structural data of recombinases prompted us to rethink how ATP hydrolysis could facilitate the removal of such unpaired roadblocks without requiring the dissociation of recombinase subunits from the nucleoprotein filament or ATP-driven rotation of DNA.

Removing the motoring requirement in the facilitated DNA-rotation model appears to be possible without eliminating the advantage of ATP hydrolysis on strand exchange (the hypothesized model is shown in Fig. 5). In this revised model, cycles of nucleotide exchange and ATP hydrolysis trigger cycles of conformational change in loop L2 and hence binding and transient release of DNA substrates. Such shakeand-bake cycles are based on the conserved cationic bridge in the ATPase centres of RadA and RecA and on the generally accepted notion that the ATP-hydrolysis cycle controls the transition of the recombinase filament between high- and low-affinity states for DNA. Homologous pairing between filament-initiating ssDNA and its homologous dsDNA appear to be much faster than the separation of the outgoing ssDNA (Bazemore, Folta-Stogniew et al., 1997; Bazemore, Takahashi et al., 1997), especially in AT-rich regions (Folta-Stogniew et al., 2004). As a result, pairing could happen intermittently between long DNA substrates. The resulting intervening dsDNA gap between paired regions (Fig. $5 a$ ) would be topologically restrained (underwound around the recombinase filament) from pairing despite its sequence homology with the filament-initiating ssDNA. Before strand exchange can proceed to completion, such a roadblock has to be removed (Shan et al., 1996; Cox, 2003; Rice et al., 2001). ATP hydrolysistriggered conformational change in $\mathrm{L} 2$ and transient release of DNA at the gap's immediate $3^{\prime}$-flank (relative to the polarity of the initiating ssDNA) would be advantageous to this requirement (Fig. 5b). As the released dsDNA gains temporary freedom, it could unwind around the recombinase filament in synchronization with proper winding of the dsDNA in the $5^{\prime}$-end of the gap (Fig. $5 c$ ). It is worth noting that such rearrangement does not change the overall topology 
of the system, thus requiring neither motor function (for active rotation) nor the dissociation of recombinase subunits (for passive rotation around a single bond in the uncoated ssDNA). As a result, pairing is enabled in the original gap region, which now becomes properly wound (Fig. $5 d$ ), and the resulting underwound dsDNA gap is displaced to the $3^{\prime}$-flank of the original one. Repetition of such displacement cycles would chase the gap out of the $3^{\prime}$-end of the entire filament, hence removing the topological roadblock to strand exchange (Fig. 5e). The directionality of gap displacement and the outcome of this revised model is essentially identical to those of the original facilitated DNA-rotation model (see Fig. 5 of Cox, 2003), except that facilitated rotation of dsDNA and synchronized ATP hydrolysis in adjacent recombinase monomers are no longer required. Since released dsDNA favours a shorter pitched B-form, the revised model appears to be compatible with the fact that recombinase filaments have shorter pitches in the absence of ATP or as a result of ATP hydrolysis (DiCapua et al., 1990; Yu et al., 2001; Story et al., 1992). The accepted 5'-to-3' direction of RecA disassembly (Shan et al., 1997; Cox et al., 2005) suggests that affinity for DNA at the $5^{\prime}$-end is lowered the most by ATP hydrolysis. In the interior of the filament, an intervening gap would create an internal pseudo $5^{\prime}$-end at the gap's $3^{\prime}$-flank. Therefore, the $5^{\prime}$ to- $3^{\prime}$ direction of gap displacement as proposed should be the preferred directionality consistent with that of strand exchange (Gupta et al., 1998). Even in the absence of a preferred direction of transient DNA release, the faster $5^{\prime}$-to$3^{\prime}$ filament-growth rate of RecA (Galletto et al., 2006) would still dictate the direction of gap displacement. Although the directionality of Rad51-promoted DNA strand exchange is an issue of debate, it is easier to comprehend a conserved polarity (Gupta et al., 1998). Since filament growth is always faster than filament disassembly, as reviewed for RecA (Roca \& Cox, 1997) and recently observed for Rad51 (van der Heijden et al., 2007; Mine et al., 2007), it is likely that the trailing $5^{\prime}$-end of the gap keeps pace with its leading $3^{\prime}$-end. RecA appears to hydrolyze ATP in synchronized unidirectional waves which traverse $\sim 120$ subunits per minute on dsDNA (Shan et al., 1997; Cox et al., 2005). If gap displacement is to follow such a wave, all gaps would be cleared within $50 \mathrm{~min}$ for a $6 \mathrm{~kb}$ dsDNA substrate. This rate estimate of gap clearance is consistent with strand-exchange experiments using $\varphi \mathrm{X} 174-$ derived DNA substrates as exemplified in a comparative study on RecA and Rad51 (Rice et al., 2001). Unlike RecA, eukaryal and archaeal homologues generally lack cooperativity and do not appear to hydrolyze ATP in a synchronized fashion. The likely sporadic nature of ATP hydrolysis by such homologues would lead to much slower gap clearance consistent with their slower rate of promoting strand exchange between $\varphi \mathrm{X} 174$ derived DNA substrates (Rice et al., 2001). The main question remains whether this model can be reconciled with the fact that DNA strand exchange takes place in the presence of nonhydrolyzable ATP analogues. Although RecA-like proteins bind DNA, they do not appear to bind short ssDNA tight enough. It is generally accepted that oligonucleotides shorter than 30 nucleotides do not fully activate the ATPase activity of RecA (Brenner et al., 1987; Bianco \& Weinstock, 1996), which can be attributed to length-dependent affinity between RecA and ssDNA (Singleton et al., 2007). Similar length-dependence may also exist for dsDNA binding. As a consequence of thermal fluctuation, short stretches of dsDNA can be temporarily released to enable gap displacement. ATP hydrolysis may simply magnify the advantage of transient release of DNA for removing topological roadblocks. The original facilitated DNA-rotation model could explain how coupled ATP hydrolysis enables extensive DNA strand exchange. By assuming a common $5^{\prime}$-to-3' directionality, our revised model could also explain extensive DNA strand exchange in the absence of ATP hydrolysis or in the presence of uncoupled ATP hydrolysis.

The research described in this paper was performed at the Canadian Light Source, which is supported by NSERC, NRC, CIHR and the University of Saskatchewan. The authors thank Dr Michel Fodje at CLS beamline 08ID-1 and Drs Gabriele Schatte and Wilson Quail at the Saskatchewan Structural Sciences Centre for assistance with the X-ray facilities. The authors also thank Xinguo Qian for her technical assistance in cloning and the strand-exchange assay. This work was supported by SHRF Establishment Grant 1425, NSERC Discovery Grant 161981-03 and CIHR and SHRF Operating Grant 63860.

\section{References}

Bacon, D. J. \& Anderson, W. F. (1988). J. Mol. Graph. 6, 219-220.

Bazemore, L. R., Folta-Stogniew, E., Takahashi, M. \& Radding, C. M. (1997). Proc. Natl Acad. Sci. USA, 94, 11863-11868.

Bazemore, L. R., Takahashi, M. \& Radding, C. M. (1997). J. Biol. Chem. 272, 14672-14682.

Bianco, P. R. \& Weinstock, G. M. (1996). Nucleic Acids Res. 24, 49334939.

Bishop, D. K., Park, D., Xu, L. \& Kleckner, N. (1992). Cell, 69, 439-456.

Brenner, S. L., Mitchell, R. S., Morrical, S. W., Neuendorf, S. K., Schutte, B. C. \& Cox, M. M. (1987). J. Biol. Chem. 262, 4011-4016.

Brünger, A. T., Adams, P. D., Clore, G. M., DeLano, W. L., Gros, P., Grosse-Kunstleve, R. W., Jiang, J.-S., Kuszewski, J., Nilges, M., Pannu, N. S., Read, R. J., Rice, L. M., Simonson, T. \& Warren, G. L. (1998). Acta Cryst. D54, 905-921.

Bugreev, D. V., Golub, E. I., Stasiak, A. Z., Stasiak, A. \& Mazin, A. V. (2005). J. Biol. Chem. 280, 26886-26895.

Bugreev, D. V. \& Mazin, A. V. (2004). Proc. Natl Acad. Sci. USA, 101, 9988-9993.

Chen, Z., Yang, H. \& Pavletich, N. P. (2008). Nature (London), 453, 489-494.

Clark, A. J. \& Margulies, A. D. (1965). Proc. Natl Acad. Sci. USA, 53, 451-459.

Conway, A. B., Lynch, T. W., Zhang, Y., Fortin, G. S., Fung, C. W., Symington, L. S. \& Rice, P. A. (2004). Nature Struct. Mol. Biol. 11, 791-796.

Courcelle, J., Ganesan, A. K. \& Hanawalt, P. C. (2001). Bioessays, 23, 463-470.

Cox, J. M., Tsodikov, O. V. \& Cox, M. M. (2005). PLoS Biol. 3, e52.

Cox, M. M. (1998). Genes Cells, 3, 65-78.

Cox, M. M. (2003). Annu. Rev. Microbiol. 57, 551-577.

Cox, M. M. (2007). Nature Rev. Mol. Cell Biol. 8, 127-138. 
Cox, M. M., Goodman, M. F., Kreuzer, K. N., Sherratt, D. J., Sandler, S. J. \& Marians, K. J. (2000). Nature (London), 404, 37-41.

Datta, S., Ganesh, N., Chandra, N. R., Muniyappa, K. \& Vijayan, M. (2003). Proteins, 50, 474-485.

Datta, S., Krishna, R., Ganesh, N., Chandra, N. R., Muniyappa, K. \& Vijayan, M. (2003). J. Bacteriol. 185, 4280-4284.

Datta, S., Prabu, M. M., Vaze, M. B., Ganesh, N., Chandra, N. R., Muniyappa, K. \& Vijayan, M. (2000). Nucleic Acids Res. 28, 49644973.

DiCapua, E., Schnarr, M., Ruigrok, R. W., Lindner, P. \& Timmins, P. A. (1990). J. Mol. Biol. 214, 557-570.

Folta-Stogniew, E., O’Malley, S., Gupta, R., Anderson, K. S. \& Radding, C. M. (2004). Mol. Cell, 15, 965-975.

Galkin, V. E., Wu, Y., Zhang, X. P., Qian, X., He, Y., Yu, X., Heyer, W. D., Luo, Y. \& Egelman, E. H. (2006). Structure, 14, 983-992.

Galletto, R., Amitani, I., Baskin, R. J. \& Kowalczykowski, S. C. (2006). Nature (London), 443, 875-878.

Gupta, R. C., Golub, E. I., Wold, M. S. \& Radding, C. M. (1998). Proc. Natl Acad. Sci. USA, 95, 9843-9848.

Heijden, T. van der, Seidel, R., Modesti, M., Kanaar, R., Wyman, C. \& Dekker, C. (2007). Nucleic Acids Res. 35, 5646-5657.

Itaya, K. \& Ui, M. (1966). Clin. Chim. Acta, 14, 361-366.

Kabsch, W. (1993). J. Appl. Cryst. 26, 795-800.

Kowalczykowski, S. C. (2000). Trends Biochem. Sci. 25, 156-165.

Kowalczykowski, S. C. \& Krupp, R. A. (1995). Proc. Natl Acad. Sci. USA, 92, 3478-3482.

Kraulis, P. J. (1991). J. Appl. Cryst. 24, 946-950.

Krishna, R., Prabu, J. R., Manjunath, G. P., Datta, S., Chandra, N. R., Muniyappa, K. \& Vijayan, M. (2007). J. Mol. Biol. 367, 1130-1144.

Lee, M. H., Chang, Y. C., Hong, E. L., Grubb, J., Chang, C. S., Bishop, D. K. \& Wang, T. F. (2005). J. Biol. Chem. 280, 40980-40984.

Liu, Y., Stasiak, A. Z., Masson, J. Y., McIlwraith, M. J., Stasiak, A. \& West, S. C. (2004). J. Mol. Biol. 337, 817-827.

Lusetti, S. L. \& Cox, M. M. (2002). Annu. Rev. Biochem. 71, 71-100.

Mazin, A. V., Zaitseva, E., Sung, P. \& Kowalczykowski, S. C. (2000). EMBO J. 19, 1148-1156.

McRee, D. E. (1999). J. Struct. Biol. 125, 156-165.

Mine, J., Disseau, L., Takahashi, M., Cappello, G., Dutreix, M. \& Viovy, J. L. (2007). Nucleic Acids Res. 35, 7171-7187.

Morimatsu, K. \& Kowalczykowski, S. C. (2003). Mol. Cell, 11, 13371347.

Pugh, B. F. \& Cox, M. M. (1988). J. Biol. Chem. 263, 76-83.

Qian, X., He, Y., Ma, X., Fodje, M. N., Grochulski, P. \& Luo, Y. (2006). J. Biol. Chem. 281, 39380-39387.
Qian, X., He, Y., Wu, Y. \& Luo, Y. (2006). J. Mol. Biol. 360, 537-547. Qian, X., Wu, Y., He, Y. \& Luo, Y. (2005). Biochemistry, 44, $13753-$ 13761.

Rajan, R. \& Bell, C. E. (2004). J. Mol. Biol. 344, 951-963.

Rice, K. P., Eggler, A. L., Sung, P. \& Cox, M. M. (2001). J. Biol. Chem. 276, 38570-38581.

Roca, A. I. \& Cox, M. M. (1997). Prog. Nucleic Acid Res. Mol. Biol. 56, 129-223.

Sandler, S. J., Satin, L. H., Samra, H. S. \& Clark, A. J. (1996). Nucleic Acids Res. 24, 2125-2132.

Saraste, M., Sibbald, P. R. \& Wittinghofer, A. (1990). Trends Biochem. Sci. 15, 430-434.

Sehorn, M. G., Sigurdsson, S., Bussen, W., Unger, V. M. \& Sung, P. (2004). Nature (London), 429, 433-437.

Seitz, E. M. \& Kowalczykowski, S. C. (2000). Mol. Microbiol. 37, 555-560.

Shan, Q., Bork, J. M., Webb, B. L., Inman, R. B. \& Cox, M. M. (1997). J. Mol. Biol. 265, 519-540.

Shan, Q., Cox, M. M. \& Inman, R. B. (1996). J. Biol. Chem. 271, 5712 5724.

Sheridan, S. D., Yu, X., Roth, R., Heuser, J. E., Sehorn, M. G., Sung, P., Egelman, E. H. \& Bishop, D. K. (2008). Nucleic Acids Res. 36, 4057-4066.

Shim, K. S., Schmutte, C., Yoder, K. \& Fishel, R. (2006). DNA Repair, 5, 718-730

Shinohara, A., Ogawa, H. \& Ogawa, T. (1992). Cell, 69, 457-470.

Singleton, S. F., Roca, A. I., Lee, A. M. \& Xiao, J. (2007). Tetrahedron, 63, 3553-3566.

Story, R. M., Weber, I. T. \& Steitz, T. A. (1992). Nature (London), 355, 318-325.

Sung, P. \& Stratton, S. A. (1996). J. Biol. Chem. 271, 27983-27986.

Tombline, G. \& Fishel, R. (2002). J. Biol. Chem. 277, 14417-14425.

VanLoock, M. S., Yu, X., Yang, S., Lai, A. L., Low, C., Campbell, M. J. \& Egelman, E. H. (2003). Structure, 11, 187-196.

Wu, Y., He, Y., Moya, I. A., Qian, X. \& Luo, Y. (2004). Mol. Cell, 15, 423-435.

Wu, Y., Qian, X., He, Y., Moya, I. A. \& Luo, Y. (2005). J. Biol. Chem. 280, 722-728.

Xing, X. \& Bell, C. E. (2004a). Biochemistry, 43, 16142-16152.

Xing, X. \& Bell, C. E. (2004b). J. Mol. Biol. 342, 1471-1485.

Yang, H., Li, Q., Fan, J., Holloman, W. K. \& Pavletich, N. P. (2005). Nature (London), 433, 653-657.

Yu, X., Jacobs, S. A., West, S. C., Ogawa, T. \& Egelman, E. H. (2001). Proc. Natl Acad. Sci. USA, 98, 8419-8424. 